\title{
Inelastic Collapse of Three Particles
}

\author{
Tong Zhou* and Leo P. Kadanoff ${ }^{\dagger}$ \\ The James Franck Institute \\ The University of Chicago \\ Chicago, IL 60637
}

(November 4, 2018)

\begin{abstract}
A system of three particles undergoing inelastic collisions in arbitrary spatial dimensions is studied with the aim of establishing the domain of "inelastic collapse" — an infinite number of collisions which take place in a finite time. Analytic and simulation results show that for a sufficiently small restitution coefficient, $0 \leq r<7-4 \sqrt{3} \approx 0.072$, collapse can occur. In one dimension, such a collapse is stable against small perturbations within this entire range. In higher dimensions, the collapse can be stable against small variations of initial conditions, within a smaller $r$ range, $0 \leq r<9-4 \sqrt{5} \approx 0.056$.
\end{abstract}

PACS: 47.50.+d, 05.20.Dd

\section{INTRODUCTION}

A system of particles interacting only through inelastic collisions is a useful idealization of granular materials, and has been much investigated recently [1] 10]. Inelasticity can make such a system evolve into a collapse state, in which several of the particles collide an infinite number of times in a finite time interval.

Inelastic collapse in one dimension is well understood [1. In two dimensions, McNamara and Young carried out numerical investigations and found some evidence for inelastic collapses of three particles [5]. To understand the collapse mechanism in higher dimensions, we study the behavior of three particles in a particular model. Our model involves collisions which preserve the total momentum and the components of the momentum perpendicular to the line of centers. The component of the relative velocity along the line of centers is reversed (as in an elastic collision) and reduced by the restitution coefficient, $0 \leq r \leq 1$. We look at a situation in which one particle (labeled zero) takes part in all collisions. The other two particles (labeled one and two) are alternatively a collider and a spectator. We assume that all particles have the same mass, and that particles one and two have identical radii.

There are two possible reasons that previous numerical studies might have shown collapse. One scenario is that the collapsed state is represented by one or many attractive fixed points, so that the collapsing orbit can be stable against small variations in initial data. The other scenario is that each orbit is unstable but that the infinity of collapsing orbits produces an observable collapse probability. For the specific example of three particles, we find an attractive fixed point for all dimensions and sufficiently small coefficient of restitution, $r$. Thus, we establish the possibility of the first scenario. The second scenario is still possible, but we have seen no evidence for it. For a larger $r$, there is an interval in which the fixed point is unstable against changes in the initial conditions.

For dimensions greater than one, after the collapse has occurred, the particles can separate from one another. Thus beyond $d=1$, inelastic collapse is an event, not an end-point, in the "lives" of the particles.

To study the collapse, we use the methods of dynamical systems theory. Specifically, we examine the situation in which particles one and two are very close to particle zero and aimed so that the system is very close to the collapsing fixed point. Figure 1 shows a typical configuration. Particle 0 keeps colliding with particle 1 and particle 2 repeatedly, and an inelastic collapse may occur. After many collisions, the distances between the particles become small, and the remaining collisions take place so rapidly that the relative motion of the particles is small. Therefore, in the inelastic collapse, the angle $\theta$ approaches a limiting value as the number of collision goes to infinity. We identify the constant- $\theta$ fixed point and then investigate its stability. We find that a fixed point of an inelastic collapse exists only when the final $\theta$ obeys:

$$
\cos \theta \geq \frac{4 \sqrt{r}}{1+r}
$$

A collapse state will occur whenever this criterion holds and also steric effect do not block off the required collisions. (For example such a blockage will always occur at $\theta=\pi$.) The stability analysis implies that for the collapse to be stable against small perturbations in the initial velocities, a stronger condition is required, namely

$$
\cos \theta>\frac{2 \sqrt[3]{r}(1+\sqrt[3]{r})}{1+r}
$$

\footnotetext{
*Email: tongzhou@control.uchicago.edu

${ }^{\dagger}$ Email: LeoP@uchicago.edu
} 


\section{THE COLLISION MODEL}

We use the standard model of inelastic collision: due to a collision the component of the relative velocity of the colliders along the line of centers, changes by a factor of $-r$. We denote by $\vec{u}_{j}$ and $\vec{x}_{j}$ the velocity and the position of the $j$ th particle at the instant before a collision occurs. Let us consider a collision between particles 1 and 0 . In the course of the collision, the velocities of the particles change to:

$$
\begin{aligned}
& \vec{u}_{1}^{\prime}=\vec{u}_{1}-\vec{\Delta} \\
& \vec{u}_{0}^{\prime}=\vec{u}_{0}+\vec{\Delta} \\
& \vec{u}_{2}^{\prime}=\vec{u}_{2}
\end{aligned}
$$

Here, the momentum transfer is $\vec{\Delta}$. It must point in the direction of the line of centers. In terms of the coefficient of restitution, $r$, this transfer is given by the expression

$$
\vec{\Delta}=\frac{1+r}{2}\left(\vec{x}_{1}-\vec{x}_{0}\right)\left[\left(\vec{x}_{1}-\vec{x}_{0}\right) \cdot\left(\vec{u}_{1}-\vec{u}_{0}\right)\right] .
$$

Here we have assumed that the radii of the colliding particles sum to unity so that, at the point of collision:

$$
\left(\vec{x}_{1}-\vec{x}_{0}\right)^{2}=1 \text {. }
$$

To do dynamical systems theory, we wish to look at the very same process repeatedly. Therefore, we introduce the superscripts, $c$, denoting the collider and $s$ denoting the spectator particle, as well as a subscript, $n$, to denote the instant before the $n$th collision occurs (Figure 1). For simplicity, we take the velocity and the position of particle 0 to be zero. In order to make sure that the velocity of particle 0 continues to vanish after the collision, we view the post-collision system from a frame moving with velocity $\vec{u}_{0}^{\prime}=\vec{\Delta}$. Then equations (3) - (5) read

$$
\begin{aligned}
& \vec{u}_{n+1}^{s}=\vec{u}_{n}^{c}-2 \vec{\Delta}, \\
& \vec{u}_{n+1}^{c}=\vec{u}_{n}^{s}-\vec{\Delta} .
\end{aligned}
$$

These equations are supplemented by the conditions:

$$
\begin{gathered}
\vec{\Delta}=\frac{1+r}{2}\left(\vec{x}_{n}^{c}\right)\left(\vec{x}_{n}^{c} \cdot \vec{u}_{n}^{c}\right), \\
\left(\vec{x}_{n}^{c}\right)^{2}=1 .
\end{gathered}
$$

Additionally, both the velocity and the position of particle 0 vanish. Notice that a collider becomes a spectator immediately following a collision. The above equations are complemented by the equations corresponding to the positions of the particles at the next collision,

$$
\begin{aligned}
& \vec{x}_{n+1}^{s}=\vec{x}_{n}^{c}+t_{n} \vec{u}_{n+1}^{s}, \\
& \vec{x}_{n+1}^{c}=\vec{x}_{n}^{s}+t_{n} \vec{u}_{n+1}^{c} .
\end{aligned}
$$

The time interval between the $n$th and the $(n+1)$ th collisions, $t_{n}$, is such that the magnitude of $\vec{x}_{n+1}^{c}$ is unity.

\section{FLAT SURFACE APPROXIMATION}

We now seek fixed points in these equations. We assume that the time between collisions is sufficiently small so that the $t_{n}$ terms in equations (9) are negligible and consequently

$$
\begin{aligned}
& \vec{x}_{n+1}^{s}=\vec{x}_{n}^{c}, \\
& \vec{x}_{n+1}^{c}=\vec{x}_{n}^{s},
\end{aligned}
$$

during the approach to the fixed point.

We wish to find a fixed point in the components of the velocity in the direction of the lines of centers. Specifically, we would like to investigate how this component decreases in each iteration. We can define:

$$
\vec{x}_{n+1}^{c} \cdot \vec{u}_{n+1}^{c} \equiv k_{n} \vec{x}_{n}^{c} \cdot \vec{u}_{n}^{c}
$$

Taking the dot product of equations (6) respectively into $\vec{x}_{n}^{s}$ and $\vec{x}_{n}^{c}$, and using equations (10) gives

$$
\begin{gathered}
\vec{x}_{n+1}^{s} \cdot \vec{u}_{n+1}^{s}=-r \vec{x}_{n}^{c} \cdot \vec{u}_{n}^{c} \\
\vec{x}_{n+2}^{c} \cdot \vec{u}_{n+2}^{c}=\vec{x}_{n+1}^{s} \cdot \vec{u}_{n+1}^{s}+\frac{1+r}{2} \vec{x}_{n+1}^{c} \cdot \vec{u}_{n+1}^{c} \cos \theta .
\end{gathered}
$$

Equations (11)-(13) thus imply the recursion satisfied by the scaling factor $k_{n}$

$$
k_{n+1}=-\frac{r}{k_{n}}+\frac{1+r}{2} \cos \theta .
$$

Fixed points can be found by setting $k_{n+1}=k_{n}$ in (14),

$$
k^{2}-k \frac{1+r}{2} \cos \theta+r=0 .
$$

As a result, the fixed point of the scaling factor has two possible values

$$
k_{ \pm}=\frac{1+r}{4} \cos \theta \pm \sqrt{\left(\frac{1+r}{4} \cos \theta\right)^{2}-r}
$$

Equation (16) is one of the major results of our study.

In every collision, the colliding particle must approach particle 0 . Hence, $\vec{x}_{c} \cdot \vec{u}_{c}$ must be negative in every iteration. This is possible only if $k$ is a positive real number. One kind of failure arises when $k$ is complex. Then the real part of the dot product will change sign infinitely often and no fixed point can be reached. Thus, for inelastic collapse to occur, the quantity under the square root in equation (16) must be positive. This positivity still permits both signs of $\cos \theta$. However, if the roots are real and the cosine is negative, both roots will be negative. Hence neither is a possible solution for inelastic collapse. The 
only remaining possibility is that inelastic collapse may occur under the condition on the cosine given by equation (11). According to that statement, when $r \rightarrow 0, \theta$ can have a value between 0 and $\frac{\pi}{2}$. On the other hand, when $\theta=0$, the well-known one-dimensional result is recovered [2], i.e., inelastic collapse is possible for $0 \leq r<7-4 \sqrt{3}$. Regions (a) and (b) in figure 2 are the region of $r$ and $\theta$ for which we may have inelastic collapse.

We now consider the stability of the above fixed points. Stability will imply that a small change in the initial conditions will leave the system in a collapse state, or in other words, changes will still permit an infinite number of collisions. There are two collapse fixed points distinguished by the values of the two multipliers $k=k_{+}$and $k=k_{-}$. Subtracting $k_{ \pm}$from both sides of (14) yields

$$
\frac{k_{n+1}-k_{ \pm}}{k_{n}-k_{ \pm}}=\frac{k_{\mp}}{k_{n}} \text {. }
$$

It is seen that $k_{-}$corresponds to an unstable fixed point, while $k_{+}$corresponds to a stable one. Henceforth, we use $k$ to denote the stable fixed point $k \equiv k_{+}$.

Next, we investigate the time interval between successive collisions. Assume that relative motion of the two colliding particles between each pair of collisions covers a distance which is very small in comparison to their radii. Then, we can think of the surface of the particles as flat. After the $n$th collision, the colliding particle moves away from the surface and covers a distance $t_{n} \vec{x}_{n+1}^{s} \cdot \vec{u}_{n+1}^{s}$. In the next step, this particle moves back over the same distance, which is given by $-t_{n+1} \vec{x}_{n+2}^{c} \cdot \vec{u}_{n+2}^{c}$ and reaches the surface. Thus, the ratio of times is

$$
\frac{t_{n+1}}{t_{n}}=\frac{-\vec{x}_{n+1}^{s} \cdot \vec{u}_{n+1}^{s}}{\vec{x}_{n+2}^{c} \cdot \vec{u}_{n+2}^{c}} .
$$

This result may be simplified with the aid of equations (11) $-(12)$ to give

$$
\frac{t_{n+1}}{t_{n}}=\frac{r}{k^{2}} .
$$

Let $d$ denote the shortest distance between two particles. The distance ratio equals the product of the time ratio and the velocity ratio, $k$,

$$
\frac{d_{n+1}}{d_{n}}=\frac{r}{k}
$$

We performed numerical simulations of the collision process by considering three inelastic particles moving in two-dimension with random initial conditions. When collapse happens, we compared the ratios calculated from simulations with the predictions of equations (11), (19), (20). We found excellent agreement (Figure 3), indicating that the fixed points are attractive and indeed correspond to collapse

\section{VALIDATION OF THE FLAT SURFACE APPROXIMATION}

As observed in the last section, making the approximation (10) simplifies dramatically the original system and it can be described by a single ratio $k_{n}$. It is as if the particles have flat surfaces, so that the effect of the tangential components of the velocities of particles 1 and 2 can be ignored. This is true only if the time intervals are negligible, so that (10) can be obtained from (9). We will see when such a simplification is valid, and we will set a criterion for the range of validity of the approximation.

In our way to equations (12)-(13), we neglected the terms like $t_{n}\left(u_{n}^{c}\right)^{2}$ in comparison to $\vec{x}_{n}^{c} \cdot \vec{u}_{n}^{c}$, by using the approximation (10). As noted above, the former terms decrease as $\left(\frac{r}{k^{2}}\right)^{n}$, while the latter terms decrease as $k^{n}$. Thus, the flat surface approximation is reliable only when $r<k^{3}$ so that terms proportional to $t_{n}$ can be safely ignored. This condition can be explicitly written as,

$$
r<\left(\frac{1+r}{4} \cos \theta+\sqrt{\left(\frac{1+r}{4} \cos \theta\right)^{2}-r}\right)^{3}
$$

which can then be simplified into the form of condition (2). The region of stability determined by this condition is region (a) in Figure 2. The maximum possible value of $r$ for stable behavior is $r_{c}=9-4 \sqrt{5}$.

To this point, our calculation did not rely on the circular geometry of the particles. The name "flat surface" suggests that when criterion (2) is satisfied, particles do not experience the curvature of their surfaces, and collide as if they are flat. This calculation is valid for arbitrary particle shapes when (2) is satisfied.

We also observe that when criterion (2) is satisfied, the time interval $t_{n}$ decreases faster than the radial component of the velocities. In such a situation, collapse happens so quickly that all other effects, external or internal, have no essential influences to the process. One can further consider arbitrary interactions between the particles as well as arbitrary external fields, as long as all the interactions depends only on the relative positions. Since the particles' relative positions only change very little during the process of collapse, all the effects of the interactions on, say, particle 1 can be replaced by a constant total force acting on it which induces an constant acceleration. This acceleration has very little effect in the tangential direction since time interval is too small for it to change the tangential component of the velocity. When the flat surface approximation is valid, the time interval is even too small to change the radial velocity component. We conclude that the the previously obtained fixed points are unchanged. 


\section{CIRCULAR GEOMETRY}

In the previous sections, the calculation were performed by neglecting the $t_{n}$ terms completely out of equations (9) and the fixed points for inelastic collapse were found when the final state satisfied condition (2). After understanding the characteristic behavior of the collapse, we can do a more rigorous calculation to investigate how the system behaves outside the region satisfying (2).

We now see that during the collapse process, the radial velocity components of particles 1 and 2 monotonically decrease till they vanish at the moment of singularity, while their tangential components approach limiting values as the number of collision diverges. Hence, we take those tangential components as constants, and concentrate on the radial components in the calculation of fixed points.

For simplicity we study in detail one collision in the situation where particle 1 has a zero radius while particle 0 has a unit radius. Since the theory depends only upon the sum of the two radii, this case subsumes all others. Here we denote the initial instant with a subscript $i$, and the instant before the collision with $f$. We drop the superscripts since only particle 1 is considered. We also assume the particles are very close, $d \ll 1$, and $-\vec{u} \cdot \vec{x}_{i} \ll u_{t}$, where $u_{t}$ is the magnitude of the tangential velocity component of particle 1 . The collision time is

$$
t=\frac{1}{u_{t}}\left(\frac{-\vec{u} \cdot \vec{x}_{i}}{u_{t}}-\sqrt{\left(\frac{\vec{u} \cdot \vec{x}_{i}}{u_{t}}\right)^{2}-2 d}\right) .
$$

Immediately before the collision, the radial velocity component of particle 1 equals

$$
-\vec{u} \cdot \vec{x}_{f}=\sqrt{\left(\vec{u} \cdot \vec{x}_{i}\right)^{2}-2 d u_{t}^{2}} .
$$

Equation (22) gives the quantity needed to complete the equation set (6)-(9). In order to abbreviate this calculation, we introduce an effective centrifugal acceleration. In the above calculation, if we view the situation in a frame rotating with an angular velocity of $u_{t}$, around an axis perpendicular to both $\vec{u}$ and the line of centers, passing through the center of particle 0 , then particle 1 has zero tangential velocity, and the effect of the tangential velocity can be represented by a centrifugal acceleration $a_{1}=u_{t}^{2}$. This substitution is justified by noticing that we can get exactly the same expressions as (22) and (23) by using this acceleration. We do not need really use such a rotating frame. We use $a_{1}$ as an effective centrifugal acceleration to replace the tangential component of the velocity with the same effects.

After these preparations, we are in a much clearer position. Particle 1 and particle 2 respectively have centrifugal accelerations $a_{1}$ and $a_{2}$, which are all in the radial directions. Equation (10) is again a good approximation. Particle 1 is moving on a line, and so is particle 2. We can further drop the vector notation. In the following, we use $u$ to denote the radial component of the velocity of particles 1 and 2 immediately before a collision, with its positive direction pointing towards the center of particle 0 . Consequently, $a_{1}=-u_{1 t}^{2}$ and $a_{2}=-u_{2 t}^{2}$.

The equation set (6)-(9) reduces to

$$
\begin{aligned}
u_{n+1}^{s} & =-r u_{n}^{c}+a_{n+1}^{s} t_{n} \\
u_{n+1}^{c} & =u_{n}^{s}+\frac{1+r}{2} \cos \theta \cdot u_{n}^{c}+a_{n+1}^{c} t_{n} \\
d_{n} & =u_{n+1}^{c} \cdot t_{n}-\frac{1}{2} a_{n+1}^{c} t_{n}^{2} \\
-d_{n+1} & =-r u_{n}^{c} \cdot t_{n}+\frac{1}{2} a_{n+1}^{s} t_{n}^{2} \\
a_{n+1}^{c} & =a_{n}^{s} \\
a_{n+1}^{s} & =a_{n}^{c}
\end{aligned}
$$

where $d_{n}$ is the distance between the spectator and particle 0 at the instant of the $n$th collision. Recall that $t_{n}$ is the time interval between the $n$th and $(n+1)$ th collisions.

One simple case can be fully carried through, the case $a_{1}=a_{2} \equiv a$. In this case, particle 1 and particle 2 are in a symmetrical situation so that the recursion relation of the system can be obtained after a single collision.

As we did before, we use two nondimensional numbers $k_{n}$ and $\alpha_{n}$ to describe the evolution of the system:

$$
k_{n} \equiv \frac{u_{n+1}^{c}}{u_{n}^{c}} \quad \text { and } \quad \alpha_{n} \equiv \frac{-a \cdot t_{n}}{u_{n}^{c}}
$$

Starting from (24), after some straightforward calculation, we get following recursion relation:

$$
k_{n+1}=\sqrt{\left(\frac{1+r}{2} \cos \theta-\frac{r}{k_{n}}\right)^{2}-(1+r) \cos \theta \frac{\alpha_{n}}{k_{n}}},
$$

and

$$
\begin{aligned}
\alpha_{n+1} & =\frac{1+r}{2} \cos \theta-\frac{r}{k_{n}}-\frac{\alpha_{n}}{k_{n}} \\
& -\sqrt{\left(\frac{1+r}{2} \cos \theta-\frac{r}{k_{n}}\right)^{2}-(1+r) \cos \theta \frac{\alpha_{n}}{k_{n}}} .
\end{aligned}
$$
have

Suppose the fixed point is $(\alpha, k)$, then from (27), we

$$
\alpha=\frac{-k^{2}+\frac{1+r}{2} \cos \theta \cdot k-r}{k+1} .
$$

Since $\alpha \geq 0$ from its definition, the right hand side of the above equality must be nonegative. Thus, there must exist two real solutions of $k$ satisfying r.h.s. $=0$. And we readily recover the condition (11).

Substituting (28) into (26), we have

$$
\begin{aligned}
k^{4}= & \left(\frac{1+r}{2} \cos \theta \cdot k-r\right)^{2} \\
& -(1+r) \cos \theta \cdot k \frac{-k^{2}+\frac{1+r}{2} \cos \theta \cdot k-r}{k+1} .
\end{aligned}
$$


Of course, $k_{+}$, which appeared before, are solutions of (29). From (28) we learn that $\left(0, k_{ \pm}\right)$are fixed points. Let us look at other solutions of equation (29) which satisfy

$$
\begin{array}{r}
k^{3}+\left(1+\frac{1+r}{2} \cos \theta\right) k^{2}-\left(r+\frac{1+r}{2} \cos \theta\right) k \\
-r=0 .
\end{array}
$$

This equation has one and only one solution of $k$ in the interval $[0: 1]$. We denote the corresponding fixed point as $\left(\alpha_{0}, k_{0}\right)$. Of the three relevant fixed points, $\left(0, k_{-}\right)$is unstable, and we concentrate on the stability condition for the other two fixed points.

Suppose that $\left(\alpha_{n}, k_{n}\right)$ has a small deviation from the fixed point $(\alpha, k)$. then

$$
\left(\begin{array}{c}
\delta k_{n+1} \\
\delta \alpha_{n+1}
\end{array}\right)=\left(\begin{array}{cc}
A_{11} & A_{12} \\
A_{21} & A_{22}
\end{array}\right)\left(\begin{array}{c}
\delta k_{n} \\
\delta \alpha_{n}
\end{array}\right)
$$

where

$$
\begin{aligned}
& A_{11}=\frac{1}{2 k^{3}}\left[2\left(\frac{1+r}{2} \cos \theta-\frac{r}{k}\right) r+(1+r) \cos \theta \cdot \alpha\right] \\
& A_{12}=-\frac{(1+r) \cos \theta}{2 k^{2}} \\
& A_{21}=\frac{\alpha+r}{k^{2}}-A_{11} \\
& A_{22}=\frac{(1+r) \cos \theta-2 k}{2 k^{2}}
\end{aligned}
$$

Let us denote by $\lambda$ the eigenvalue of matrix $A$, hence we have

$$
\lambda^{2}-b \lambda+c=0
$$

where

$$
\begin{aligned}
b= & \frac{1}{2}-\frac{1}{k}+\left[(1+r) \cos \theta-\left(\frac{1+r}{2} \cos \theta\right)^{2}\right] \frac{1}{2 k^{2}} \\
& +r(1+r) \cos \theta \cdot \frac{1}{k^{3}}-\frac{3 r^{2}}{2 k^{4}} \\
c= & \frac{r^{2}}{k^{5}}
\end{aligned}
$$

For the fixed point $\left(0, k_{+}\right)$,

$$
b=\frac{r}{k_{+}^{3}}\left(1+k_{+}\right) \quad \text { and } \quad c=\frac{r^{2}}{k_{+}^{5}} .
$$

So

$$
\lambda_{1}=\frac{r}{k_{+}^{2}} \quad \text { and } \quad \lambda_{2}=\frac{r}{k_{+}^{3}} .
$$

The point $\left(0, k_{+}\right)$is an attractive fixed point if and only if $r<k_{+}^{3}$. Hence we recover the condition of (2). If we only require $\lambda_{1}<1$, we can recover condition (11).

For the fixed point $\left(\alpha_{0}, k_{0}\right)$, we find that $\alpha_{0}>0$ if and only if the condition of (2) is satisfied, but that condition also decides the range of $(r, \theta)$ inside which we will have

$$
1-b+c<0
$$

From equation (32) we know that one eigenvalue of matrix $A$ is larger than 1, implying that the corresponding fixed point is unstable. We conclude that there are no additional stable fixed points beyond those which satisfy condition (2), and there are no stable collapses outside that range in the case $a_{1}=a_{2}$.

We believe this is true for the general situation of $a_{1} \neq a_{2}$. When condition (2) is violated, our simulations show that even though the particles can be very close, they will get apart before having collided an infinite number of times.

From the above calculation, and specifically equation (34), we can see the parameter space $(r, \theta)$ can be divided into three regions (Figure 2):

a) When condition (2) is satisfied, both the eigenvalues of matrix $A$ are smaller than unity. The fixed point is stable in all directions in space $(\alpha, k)$. It is the collapse region.

b) When condition (2) is violated but the condition (11) is satisfied, the eigenvalue $\lambda_{2}>1$, while $\lambda_{1}<1$. In this region, particles can have any number of collisions before they might eventually separate.

c) When the condition (11) is violated, both eigenvalues of matrix $A$ are larger than unity. Collapse does not occur.

The above calculation is independent of the sources of the accelerations, which could be the interactions between particles. The calculation confirms our previous argument that interactions are irrelevant in the process of collapse ${ }^{1}$.

We also observe that in the above calculation, the circular geometry of the particles is not essential. The calculation is also valid for arbitrary shape of the particles, with the corresponding radius of curvature replacing the radii used. Even though the radius of curvature is not

\footnotetext{
${ }^{1}$ But when there is a sufficiently strong attraction, e. g. gravity, between particles so that the directions of the accelerations are reversed, we want fixed points with $\alpha \leq 0$. Then the fixed point $\left(\alpha_{0}, k_{0}\right)$ becomes stable when condition (2) is violated. Inelastic collapse can happen in a much larger region of $(r, \theta)$.
} 
relevant in deciding the behavior of collapse process - the radii do not show up in the expression of the stable fixed point or condition (2), it does have some effects. The centrifugal acceleration, which obviously is important in deciding the probability of collapse, is inversely proportional to the radius. So chances are larger for collapse to happen when the colliding point is at a position on the surface with a larger radius of curvature.

\section{CONCLUSION}

We demonstrated analytically the existence of inelastic collapse for three particles in all dimensions. At the last moment of collapse, the three particles have a cyclic behavior, which is characterized by a fixed point. We have established the range of the parameters for which the fixed point exists and the range for which it is stable.

\section{Acknowledgments}

We would like to express our thanks to Y.Du, S. Esipov, H. M. Jaeger, M. Mungan, S. R. Nagel, N. Schörghofer and W. R. Young for very helpful discussions and especially to Eli Ben-Naim for continued advice and discussions. This work was supported in part by NSF-DMR and in part by DOE.

[1] B. Bernu and R. Mazighi, J. Phys. A 23, 5745 (1990).

[2] S. McNamara and W. R. Young, Phy. Fluids A 4, 496 (1992).

[3] E. Clement, S. Luding, A. Blumen, J. Rajchenbach and J. Duran, Int. J. Mod. Phys. B 7, 1807 (1993).

[4] P. Constantin, E. Grossman and M. Mungan, Physica D 83, 409 (1995).

[5] S. McNamara and W. R. Young, Phy. Rew. E 50, R28 (1994).

[6] P. K. Haff, J. Fluid Mech. 134, 401 (1983).

[7] M. A. Hopkins and M. Y. Louge, Phy. Fluids A 3, 4 (1990).

[8] I. Goldhirsch and G. Zanetti, Phys. Rev. Lett. 70, 1619 (1993).

[9] Y. Du, H. Li and L. P. Kadanoff, Phys. Rev. Lett. 74, 1268 (1995).

[10] H. M. Jaeger, S. R. Nagel and R. P. Behringer, "The Physics of Granular Materials", preprint. 